\title{
What determines the emission peak energy of the blue luminescence in highly Mg-doped $\mathrm{p}-\mathrm{GaN}$ ?
}

John S. Colton

john_colton@byu.edu

P. Y. Yu

Follow this and additional works at: https://scholarsarchive.byu.edu/facpub

Part of the Astrophysics and Astronomy Commons, and the Physics Commons

\section{Original Publication Citation}

What determines the emission peak energy of the blue luminescence in highly Mg-doped pGaAs? J.S. Colton and P.Y. Yu, Appl. Phys. Lett. 78, 25 (21). The original version may be found at: http://apl.aip.org/resource/1/applab/v78/i17/p25_s1

\section{BYU ScholarsArchive Citation}

Colton, John S. and Yu, P. Y., "What determines the emission peak energy of the blue luminescence in highly Mg-doped p-GaN?" (2001). Faculty Publications. 575.

https://scholarsarchive.byu.edu/facpub/575

This Peer-Reviewed Article is brought to you for free and open access by BYU ScholarsArchive. It has been accepted for inclusion in Faculty Publications by an authorized administrator of BYU ScholarsArchive. For more information, please contact ellen_amatangelo@byu.edu. 


\title{
AIP Applied Physics \\ Letters
}

\section{What determines the emission peak energy of the blue luminescence in highly Mg-doped p-GaN?}

\author{
J. S. Colton and P. Y. Yu
}

Citation: Appl. Phys. Lett. 78, 2500 (2001); doi: 10.1063/1.1367904

View online: http://dx.doi.org/10.1063/1.1367904

View Table of Contents: http://apl.aip.org/resource/1/APPLAB/v78/i17

Published by the American Institute of Physics.

\section{Related Articles}

Laplace deep level transient spectroscopy of electron traps in epitaxial metalorganic chemical vapor deposition grown n-GaSb

J. Appl. Phys. 113, 024505 (2013)

Defect assistant band alignment transition from staggered to broken gap in mixed As/Sb tunnel field effect transistor heterostructure

J. Appl. Phys. 112, 094312 (2012)

Tuning the binding energy of surface impurities in cylindrical GaAs/AIGaAs quantum dots by a tilted magnetic field

J. Appl. Phys. 112, 064326 (2012)

Binding energies and oscillator strengths of impurity states in wurtzite InGaN/GaN staggered quantum wells J. Appl. Phys. 112, 053525 (2012)

Analytical modeling of bare surface barrier height and charge density in AIGaN/GaN heterostructures Appl. Phys. Lett. 101, 103505 (2012)

\section{Additional information on Appl. Phys. Lett.}

Journal Homepage: http://apl.aip.org/

Journal Information: http://apl.aip.org/about/about_the_journal

Top downloads: http://apl.aip.org/features/most_downloaded

Information for Authors: http://apl.aip.org/authors

\section{ADVERTISEMENT}

\section{AIP Applied Physics Letters}

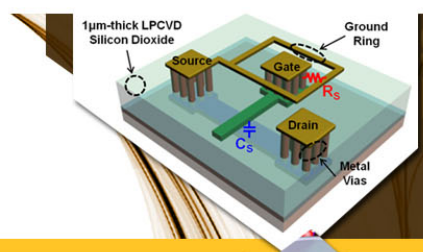

\section{SURFACES AND} INTERFACES

Focusing on physical, chemical, biological structural, optical, magnetic and electrical properties of surfaces and interfaces, and more..

\section{EXPLORE WHAT'S NEW IN APL}

SUBMIT YOUR PAPER NOW!
ENERCY CONVERSION AND STORACE 


\title{
What determines the emission peak energy of the blue luminescence in highly Mg-doped $p$-GaN?
}

\author{
J. S. Colton and P. Y. Yu ${ }^{a)}$ \\ Department of Physics, University of California, Berkeley, California 94720 and Materials Sciences \\ Division, Lawrence Berkeley National Laboratory, Berkeley, California 94720
}

(Received 27 December 2000; accepted for publication 28 February 2001)

\begin{abstract}
We report a study of the $2.8 \mathrm{eV}$ blue luminescence (BL) in heavily $\mathrm{Mg}$-doped $p$-GaN via resonant excitation with a tunable blue dye laser. The dependence of the BL on the excitation photon energy $\left(E_{\mathrm{ex}}\right)$ is unlike that of the yellow luminescence found in $n$-type GaN. An Urbach-type band tail, with Urbach parameter of $33 \mathrm{meV}$ is observed in the vicinity of the BL energy. We propose that the peak energy of the BL marks the transition from localized states to delocalized states within this band tail.

(C) 2001 American Institute of Physics. [DOI: 10.1063/1.1367904]
\end{abstract}

GaN is a wide-band-gap III-V semiconductor with applications such as light emitting diodes and laser diodes. ${ }^{1}$ One challenge in the fabrication of GaN diodes has been the achievement of a high concentration of holes. The acceptors of highly doped $p$-type $\mathrm{GaN}$ are often compensated. ${ }^{2,3}$ Accompanying this compensation is the appearance of a broad blue luminescence $(\mathrm{BL})$ centered around $2.8 \mathrm{eV}{ }^{4}$ The origin of the BL has remained controversial. Most groups working on this problem agree that the BL involves a donor-acceptor pair (DAP) transition. ${ }^{3-9}$ However, the donors and the acceptors are assumed to be shallow in some models and deep in others. Some models emphasize the importance of potential fluctuations caused by ionized impurities. ${ }^{10,11}$ So far there has been no satisfactory explanation as to what determines the peak energy of this emission. In this letter we report a study of the BL via resonant excitation with a tunable laser. We found that the dependence of the BL on the excitation photon energy $\left(E_{\mathrm{ex}}\right)$ is quite unlike that of the yellow luminescence (YL) found in $n$-type GaN. ${ }^{12}$ First, the BL intensity increases exponentially as a function of $E_{\mathrm{ex}}$ indicating the existence of an Urbach tail in the absorption edge of $p-\mathrm{GaN}$. The emission peak energy $\left(E_{\mathrm{em}}\right)$ depends on $E_{\mathrm{ex}}$ like Raman scattering (RS) for $E_{\mathrm{ex}}<2.8 \mathrm{eV}$ but changes to a behavior more like photoluminescence (PL) for $E_{\mathrm{ex}}>2.8 \mathrm{eV}$. To explain these results we propose that emission peak energy of the BL represents the energy where the photoexcited carrier lifetime changes from being dominated by relaxation to being dominated by radiative recombination. The peak of the BL is an analog of the "bottleneck" in the decay of exciton polaritons. ${ }^{13}$

Our experiment was performed with a Stilbene 420 dye laser pumped by an $\mathrm{UV} \mathrm{Ar}^{+}$-ion laser. The "normal" $\mathrm{BL}$ (i.e., BL excited by above-band-gap photons) from our $\mathrm{GaN}$ sample was excited by a $\mathrm{HeCd}$ laser at $3.814 \mathrm{eV}$. The sample was maintained at $12 \mathrm{~K}$ by a closed-cycle helium refrigerator. The BL signal was analyzed with a SPEX double spectrometer and detected with a cooled GaAs photomultiplier tube. The sample was grown via metalorganic chemical vapor deposition (MOCVD) on a sapphire substrate. It was annealed at $750{ }^{\circ} \mathrm{C}$ for $\sim 4$ min after growth and has a room

${ }^{a)}$ Electronic mail: pyyu@lbl.gov temperature carrier concentration of $p=2 \times 10^{17} \mathrm{~cm}^{-3}$, which is well below the $\mathrm{Mg}$ concentration $\left(>10^{19} \mathrm{~cm}^{-3}\right)$ introduced during growth.

In Fig. 1 we compare the "normal" BL spectrum of our sample (which is similar to those reported in the literature) with selectively excited spectra. Small oscillations are noticeable in all spectra as a result of Fabry-Perot interference occurring in the GaN film. The selectively excited BL exhibited little reduction in width when compared to the "normal" BL. The selectively excited BL showed a shift in $E_{\mathrm{em}}$ with $E_{\text {ex }}$. A plot of $E_{\text {em }}-E_{\text {ex }}$ vs $E_{\text {ex }}$ is shown in Fig. 2. We find this dependence to be separable into two regimes: (1) For $E_{\mathrm{ex}}<\sim 2.8 \mathrm{eV}$, the energy $E_{\mathrm{ex}}-E_{\mathrm{em}}$ was approximately constant, as in RS, and $\sim 180 \mathrm{meV}( \pm 10 \mathrm{meV})$. (2) For $E_{\mathrm{ex}}>\sim 2.8 \mathrm{eV}, E_{\mathrm{em}}$ varied linearly with $E_{\mathrm{ex}}$, as is typical in PL. However the slope of $E_{\mathrm{em}}-E_{\mathrm{ex}}$ vs $E_{\mathrm{ex}}$ is $-0.43( \pm 0.05)$, rather than -1 for normal PL (where $E_{\text {em }}$ would be a constant). To highlight the unusual nature of BL (shown by a dashed line for $E_{\text {ex }}>2.8 \mathrm{eV}$ ), in Fig. 2 we have indicated the dependence of normal PL and RS by dotted and solid lines, respectively. Another unusual feature of the selectively excited $\mathrm{BL}$ is the exponential dependence of its intensity $\left(I_{\mathrm{BL}}\right)$ on $E_{\mathrm{ex}}: I_{\mathrm{BL}} \sim \exp \left(E_{\mathrm{ex}} / E_{0}\right)$, where $E_{0}=33 \mathrm{meV}( \pm 5 \mathrm{meV})$ as shown in the inset of Fig. 2. This behavior is reminiscent of

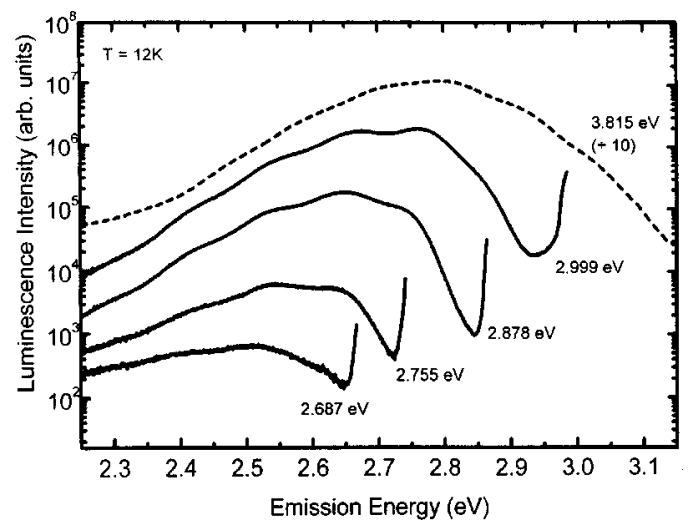

FIG. 1. "Normal"' BL (dashed curve) excited at 3.815 eV energy, compared to representative selectively excited BL (solid curves) excited at various photon energies, on a $\log _{10}$ scale. The excitation energy is shown next to each spectrum. In all cases the sample temperature was $12 \mathrm{~K}$. 


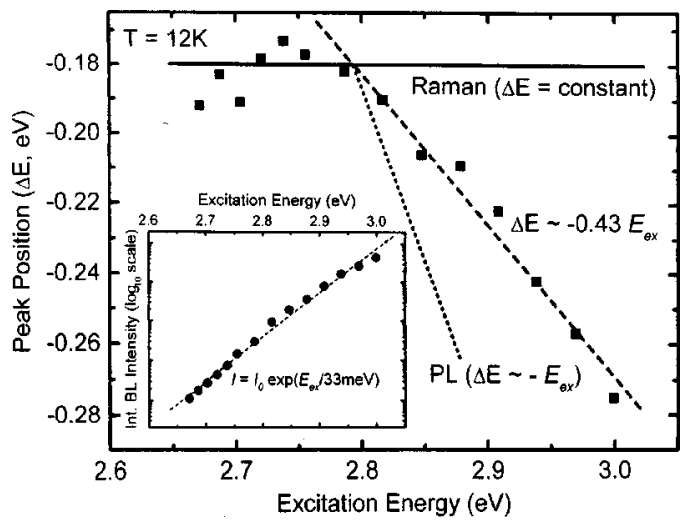

FIG. 2. Peak positions of selectively excited BL (shown as $E_{\mathrm{em}}-E_{\mathrm{ex}}$ ) plotted as a function of excitation energy $E_{\text {ex }}$. The dashed line is a linear fit to data for $E_{\mathrm{ex}}>2.8 \mathrm{eV}$. The dotted and solid lines represent the usual dependence on $E_{\mathrm{ex}}$ expected for normal PL and RS, respectively. The inset shows the integrated intensity of the selectively excited BL peaks plotted as a function of $E_{\mathrm{ex}}$, on a $\log _{10}$ scale. The solid line is a linear least-squares fit of the data with the expression: $I_{\mathrm{BL}} \sim \exp \left(E_{\mathrm{ex}} / E_{0}\right)$ with $E_{0}=33 \mathrm{meV}$. The sample temperature was $12 \mathrm{~K}$.

Urbach tails in the absorption edges of highly disordered materials.

The exponential dependence of $I_{\mathrm{BL}}\left(E_{\mathrm{ex}}\right)$ can be understood if we assume that $I_{\mathrm{BL}}$ is proportional to the absorption coefficient of the GaN sample at $E_{\text {ex }}$. Observation of Urbach tails has previously been reported by Qiu et al. in both $n$ - and p-type GaN using photoconductivity spectroscopy. ${ }^{14}$ They determined Urbach parameters $\left(E_{0}\right)$ of $180-280 \mathrm{meV}$ in both types of $\mathrm{GaN}$ samples. In an unintentionally doped $n$-type sample they observed an additional Urbach tail nearer the band gap with Urbach parameter of $\sim 50 \mathrm{meV}$. They suggested that both tails existed in all samples, with the narrower tail near the band edges being mainly due to potential fluctuations introduced by dopants, and the broader one, which extended well below $2 \mathrm{eV}$, tentatively attributed to "defect states." Thus our measured value of $E_{0}$ is quite consistent with the Urbach parameter attributed to potential fluctuations by Qiu et al.

The exponential and continuous density of states indicated by the band tail in highly doped $p$-type GaN makes it difficult to understand what determines the BL peak energy. Figure 2 suggests that the $2.8 \mathrm{eV}$ peak energy of the $B L$ is the energy where the $B L$ undergoes a transformation from $R S$ to $P L$ (we will address the deviation of the slope from -1 in the $\mathrm{PL}$ regime later). We note that the RS behavior of the $\mathrm{BL}$ is similar to that of the YL and can be understood by assuming that the selectively excited DAP states recombine without energy relaxation. In addition, the Raman shift $\left(E_{\mathrm{ex}}-E_{\mathrm{em}}\right)$ of $\sim 180 \mathrm{meV}$ is approximately equal to the energy of two longitudinal optical (LO) phonons in GaN. The absence of spectral narrowing when the BL is selectively excited suggested that the BL is homogeneously broadened by strong coupling between the LO phonons and the DAP involved in the recombination. This result is in good agreement with the conclusion of Kaufmann et al. ${ }^{15}$ who independently deduced (from their analysis of the BL line shape) a Frank-Condon shift of $180 \mathrm{meV}$. It is generally assumed that PL, unlike RS, involves energy relaxation of the photoexcited electrons and holes before radiative recombination. The fact that the $\mathrm{BL}$ changes from RS to behaving like PL for $E_{\mathrm{ex}}>2.8 \mathrm{eV}$ therefore indicates that DAP excited above this energy can relax to lower energy states.

Since $2.8 \mathrm{eV}$ is also the energy of the BL emission peak, one can understand this seeming coincidence with the following model. Let us assume that there is a strong increase with energy in the rate of carriers hopping between impurities (a relaxation rate) in the Urbach tail. This is reasonable since carriers with higher energy are expected to have higher probability of hopping into lower energy states. The recombination rate due to tunneling between donors and acceptors should also strongly increase with energy, since the higher energy photons are emitted by spatially closer DAPs. However, if we assume that these two rates differ in their dependence on energy, then it is possible that the relaxation rate can be lower than the recombination rate at low energies but becomes higher at a well-defined energy, $E_{C}$. Under this assumption, the emission should behave like RS for $E_{\mathrm{em}}$ $<E_{C}$ since photoexcited carriers recombine before relaxation. For $E_{\mathrm{em}}>E_{C}$ carriers have time to relax before recombination and therefore the emission should behave more like PL. Furthermore, the emission will peak at $E_{C}$ for $E_{\mathrm{em}}$ $>E_{C}$ since carriers will tend to relax towards to $E_{C}$.

We note that this model bears similarity to the excitonpolariton "bottleneck." 13 Although the density of states of exciton polariton are known to be continuous, the polariton emission shows usually one to two peaks whose energies do not necessarily coincide with the exciton energy. It was pointed out by Toyozawa that the polariton emission would peak at energies where the exciton-polariton lifetime was maximum (the bottleneck). ${ }^{16}$ Since the dependence of recombination rate on carrier energy within a DAP transition model is exponential, ${ }^{17}$ our model is plausible only if the dispersion in the relaxation time of carriers is even greater. Strong dispersion in the hopping time of carriers has been reported in amorphous and glassy semiconductors in the vicinity of a "mobility edge." The corresponding energy separation between the mobility edges for electrons and holes is known as the "mobility gap." We, therefore, suggest that the peak energy of $\sim 2.8 \mathrm{eV}$ for the BL is determined similarly by the energy of a "mobility gap" in the band tails which gives rise to a bottleneck in the relaxation. In principle, this bottleneck in GaN could be observed by energy dependent time-resolved photoluminescence (TRPL). For emission with $E_{\mathrm{em}}<2.8 \mathrm{eV}$, the decay time will be dominated by slow radiative recombination of localized carriers. For $E_{\mathrm{em}}>2.8 \mathrm{eV}$, the carriers are delocalized and their decay time becomes very fast as a result of relaxation into lower energy states. Energy-resolved TRPL studies have not been reported for the BL in GaN. It was, however, mentioned in a recent TRPL study of the BL that the decay time "is more strongly energy position dependent than the yellow luminescence." 18

Finally, in our model we will explain the observed relationship of $E_{\mathrm{em}}-E_{\text {ex }}$ approximately $-0.43 E_{\mathrm{ex}}$ which seems to suggest that the carriers do not relax all the way down to the bottleneck as proposed above. We first note that several groups have reported blueshifts in the DAP emission with increasing excitation intensity $I_{\mathrm{ex}}$. For example, Kuskovsky et al., found a nearly linear dependence of the DAP emission peak position on $\log _{10} I_{\text {ex }}$ in heavily $n$-doped ZnSe. ${ }^{19}$ Similar 
dependence has been reported for the BL by Reshchikov et al. ${ }^{5}$ and by Kaufmann et al. ${ }^{6}$ whose data indicate values of $d E_{\mathrm{em}} / d\left(\log _{10} I_{\mathrm{ex}}\right)$ of $\sim 57$ and $82 \mathrm{meV}$ per intensity decade, respectively. From similar measurements on our GaN sample using above band gap excitation we obtained $d E_{\mathrm{em}} / d\left(\log _{10} I_{\mathrm{ex}}\right)=72 \pm 8 \mathrm{meV}$. Kuskovsky et al. explained their results via a model in which photoexcited carriers screened the potential fluctuations introduced by dopants and therefore produced a blueshift in $E_{\text {em }}$ with carrier density $N$. We can explain why $E_{\mathrm{em}}-E_{\mathrm{ex}}$ approximately $-0.43 E_{\mathrm{ex}}$ if we assume that $N$ is proportional to $\exp \left(E_{\mathrm{ex}} / E_{0}\right)$ as a result of the Urbach tail in the below-band-gap absorption. Combining this assumption with a linear dependence of $E_{\mathrm{em}}$ on $\log _{10} N$ then implies a linear dependence of $E_{\mathrm{em}}$ on $E_{\mathrm{ex}}$, rather than a $E_{\text {em }}$ which is independent of $E_{\text {ex }}$ as in normal PL. In fact from the -0.43 slope (from the peak position dependence) combined with the measured $E_{0}$ (from the intensity dependence), we calculate that $d E_{\mathrm{em}} / d\left(\log _{10} N\right)$ $\sim 43 \mathrm{meV}$. This value is smaller than the directly measured value of $d E_{\mathrm{em}} / d\left(\log _{10} I_{\mathrm{ex}}\right)=72 \mathrm{meV}$, which may possibly be explained by noting that in the $E_{\mathrm{em}}$ vs $I_{\mathrm{ex}}$ experiment carriers are excited by above-band-gap photons while in the $E_{\text {em }}$ vs $E_{\text {ex }}$ experiment the carriers are excited by below-band-gap photons. The more energetic carriers in the former experiment should be more effective in screening the potential fluctuations and produce a larger value for $d E_{\mathrm{em}} / d\left(\log _{10} N\right)$.

In conclusion, we have interpreted the results of our experiment on selectively excited $\mathrm{BL}$ in highly $\mathrm{Mg}$-doped $\mathrm{GaN}$ within a model of DAP recombination which includes two features: (1) a large potential fluctuation due charged donors and acceptors which gives rise to an Urbach tail and a blueshift of the emission energy with photoexcited carrier density; and (2) a sharp transition from localized states to delocalized states occurring within the Urbach tail at the BL peak energy of $2.8 \mathrm{eV}$.
This work was supported by the Director, Office of Energy Research, Office of Basic Energy Sciences, Materials Sciences Division, of the U.S. Department of Energy under Contract No. DE-AC03-76SF00098.

${ }^{1}$ See, for example, S. Nakamura and G. Fasol, The Blue Laser Diode (Springer, New York, 1997).

${ }^{2}$ W. Götz, N. M. Johnson, J. Walker, D. P. Bour, and R. A. Street, Appl. Phys. Lett. 68, 667 (1996).

${ }^{3}$ L. Eckey, U. von Gfug, J. Holst, A. Hoffmann, A. Kaschner, H. Siegle, C. Thomsen, B. Schineller, K. Heime, M. Heuken, O. Schön, and R. Beccard, J. Appl. Phys. 84, 5828 (1998).

${ }^{4}$ H. Obloh, K. H. Bachem, U. Kaufmann, M. Kunzer, M. Maier, A. Ramakrishnan, and P. Schlotter, J. Cryst. Growth 195, 270 (1998).

${ }^{5}$ M. A. Reshchikov, G. C. Yi, and B. W. Wessels, Phys. Rev. B 59, 13176 (1999).

${ }^{6}$ U. Kaufmann, M. Kunzer, M. Maier, H. Obloh, A. Ramakrishnan, B. Santic, and P. Schlotter, Appl. Phys. Lett. 72, 1326 (1998).

${ }^{7}$ J. K. Sheu, Y. K. Su, G. C. Chi, B. J. Pong, C. Y. Chen, C. N. Huang, and W. C. Chen, J. Appl. Phys. 84, 4590 (1998).

${ }^{8}$ Y.-H. Kwon, S. K. Shee, G. H. Gainer, G. H. Park, S. J. Hwang, and J. J. Song, Appl. Phys. Lett. 76, 840 (2000).

${ }^{9}$ F. Shahedipour and B. W. Wessels, Appl. Phys. Lett. 76, 3011 (2000).

${ }^{10}$ E. Oh, H. Park, and Y. Park, Appl. Phys. Lett. 72, 70 (1998).

${ }^{11}$ E. R. Glaser (private communication).

${ }^{12}$ J. S. Colton, P. Y. Yu, K. L. Teo, P. Perlin, E. R. Weber, I. Grzegory, and K. Uchida, Physica B 273-274, 75 (1999); J. S. Colton, P. Y. Yu, K. L. Teo, E. R. Weber, P. Perlin, I. Grzegory, and K. Uchida, Appl. Phys. Lett. 75, 3273 (1999).

${ }^{13}$ See, for example, P. Y. Yu and M. Cardona, Fundamentals of Semiconductors, 2nd ed. (Springer, Berlin, 1999), p. 353.

${ }^{14}$ C. H. Qiu, C. Hoggatt, W. Melton, M. W. Leksono, and J. I. Pankove, Appl. Phys. Lett. 66, 2712 (1995).

${ }^{15}$ U. Kaufmann, M. Kunzer, H. Obloh, M. Maier, C. Manz, A. Ramakrishnan, and B. Santic, Phys. Rev. B 59, 5561 (1999).

${ }^{16}$ Y. Toyozawa, Suppl. Prog. Theor. Phys. 12, 111 (1959).

${ }^{17} \mathrm{P} . \mathrm{Y}$. Yu and M. Cardona, Fundamentals of Semiconductors, 2nd ed. (Springer, Berlin, 1999), p. 349.

${ }^{18}$ Y.-H. Kwon, S. KI. Shee, G. H. Gainer, G. H. Park, S. J. Hwang, and J. J. Song, Appl. Phys. Lett. 76, 840 (2000).

${ }^{19}$ I. Kuskovsky, D. Li, G. F. Neumark, V. N. Bondarev, and P. V. Pikhitsa, Appl. Phys. Lett. 75, 1243 (1999). 